\title{
A INCUBAC̣̃̃O DE OVOS E LARVAS EM GENIDENS GENIDENS (VALENCIENNES) (SILURIFORMES, ARIIDAE) DA BAÍA DE GUARATUBA, PARANÁ, BRASIL ${ }^{1}$
}

\author{
Paulo de Tarso da C. Chaves ${ }^{2}$
}

\begin{abstract}
Eggs and larvaE mouthbreeding in Genidens genidens (Valenciennes) (Siluriformes, AriIdae) from Guaratuba Bay, ParanÁ, Brazil. At Guaratuba bay, the catfish Genidens genidens shows incubating behavior during the summer months. The larvae are either less or heavier than the eggs, and their stomach analysis confirms the exogenous feeding during incubation. Embryos sizes can increase to $59 \mathrm{~mm}$ in total lenght and $2,8 \mathrm{~g}$ in total weight, including the yolksac. It was observed that the largest larvae are guarded by the largest adults, and that the embryo size is similar among individuals from the same adult.
\end{abstract}

KEY WORDS. Catfish, reproduction, fishes, larvae, Paraná

Os bagres da familia Ariidae são típicos incubadores orofaringeais de ovos e larvas. A função é normalmente desempenhada pelos machos (BREEDER \& ROSEN 1966), e em algumas espécies o período de incubação pode ultrapassar dois meses (OPPENHEIMER 1970). As fềmeas de maior porte produzem ovócitos de maior tamanho (COATES 1988) e em maior número (ETCHEVERS 1978; Mishima \& TANJI 1985) que as menores. Quanto aos machos, também são os indivíduos maiores que conduzem maior quantidade de ovos e de embriões (BARBIERI et al. 1992), bem como os embriões de maior porte (COATES 1988).

Outras informações disponíveis acerca da reprodução em espécies deste grupo dizem respeito a atributos populacionais, como local, época e tipo de desova, e ainda tamanho de primeira maturação, estudados na costa da Venezuela por ETChevers (1978), no litoral sul de São Paulo por Mishima \& TANJi (1985), na Lagoa dos Patos (Rio Grande do Sul) por ReIs (1986), em Papua Nova Guiné por COATES (1988), e na lagoa de Jacarepaguá (Rio de Janeiro) por BARBIERI et al. (1992). Na foz do rio Itajaí-Açu, litoral norte de Santa Catarina, estudo de populações locais de Ariidae também vem sendo realizado (MAURícıo HostiMSILVA, comun. pess.). É ressaltada por vários destes trabalhos a importância de um correto manejo pesqueiro com os bagres, visto que as peculiaridades em seu comportamento reprodutivo os torna muito dependentes da densidade populacional.

1) Contribuição número 804 do Departamento de Zoologia, Universidade Federal do Paraná. Auxilios CNPq e FUNPAR.

2) Departamento de Zoologia, Universidade Federal do Paraná, Caixa Postal 19020, 81531-970 Curitiba, Paraná, Brasil. Pesquisador do CNPq. 
Em recente pesquisa sobre a reprodução de peixes na baía de Guaratuba, litoral do Paraná, chamou a atenção o fato de em Genidens genidens (Valenciennes, 1839) o peso das larvas incubadas (e ainda dotadas de reserva vitelínica) atingir valores bem mais altos que aqueles dos ovos. De fato, em peixes vivíparos é esperado um aumento de peso ao longo do desenvolvimento embrionário, graças a contribuições de secreções uterinas. Mas, em espécies ovulíparas, geralmente a obtenção de alimento do meio externo só tem início após o esgotamento, ou pelo menos significativa redução, do saco vitelínico (PEÑa et al. 1988). A partir daí as larvas, mesmo incubadas, podem efetuar alimentação exógena, inclusive de secreções da câmara incubadora (OPPENHEIMER 1970). Assim, este trabalho investiga a hipótese de que as larvas de $G$. genidens, simultaneamente à utilização do saco vitelínico, consomem alimento de origem externa. Paralelamente, objetiva contribuir à biologia reprodutiva da espécie na região, descrevendo alguns aspectos de sua dinâmica de incubação.

\section{MATERIAL E MÉTODOS}

Exemplares de $G$. genidens em reprodução foram coletados com rede de arrasto de fundo, com portas, em janeiro e fevereiro de 1994, nos canais de mangue

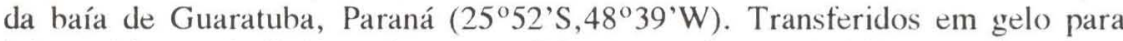
laboratório, os indivíduos foram medidos (comprimento total) e pesados. Examinada a cavidade orofaringeana, sempre que presentes foram retirados e fixados em formol $10 \%$ os ovos ou larvas. Muitas larvas também foram recolhidas diretamente da rede, possivelmente por terem durante a pesca abandonado seus abrigos. No total, foram analisados 47 ovos embrionados e 52 larvas. Em 22 adultos incubadores foi feita ainda uma incisão ventral, objetivando visualização das gônadas e do trato digestivo.

Atividade laboratorial posterior com os ovos e as larvas, utilizando balança com precisão de $0,1 \mathrm{~g}$ e paquímetro, consistiu em tomadas individuais das seguintes informações: maior diâmetro do ovo, peso do ovo, comprimento total da larva (focinho à extremidade distal da cauda) e peso da larva (incluindo embrião e saco vitelínico).

A cavidade abdominal de 10 larvas (comprimento entre 33,2 e $52,8 \mathrm{~mm}$ ) foi dissecada para análise do conteúdo estomacal em microscópio.

\section{RESULTADOS}

Os ovos embrionados apresentaram peso entre 1,5 e $1,9 \mathrm{~g}$, e maior diâmetro entre 13,1 e $15,5 \mathrm{~mm}$. As larvas, por seu turno, apresentaram-se tanto mais leves como mais pesadas, entre 0,8 e $2,8 \mathrm{~g}$, e seu comprimento variou entre 32,0 e $59,0 \mathrm{~mm}$. Observou-se que, assim como ovos de maior diâmetro possuem maior peso (Fig. 1), também o peso das larvas cresce à medida que seu comprimento aumenta (Fig. 2).

Todas as larvas encontradas retinham maior ou menor volume de saco vitelínico (Fig. 3). Algumas que ainda a bordo foram colocadas em vidro com 


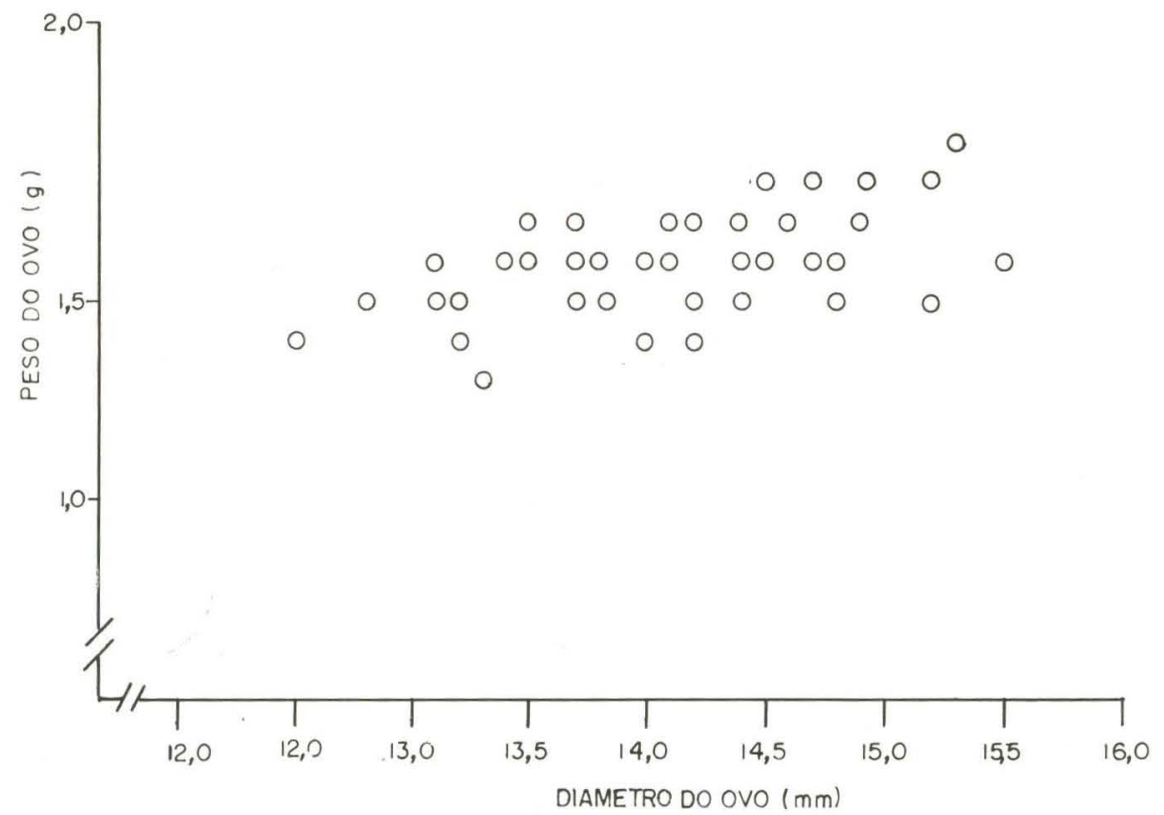

Fig. 1. Relação entre os valores de peso e de maior diâmetro nos ovos de Genidens genidens. Número total de ovos mensurados: 46 .

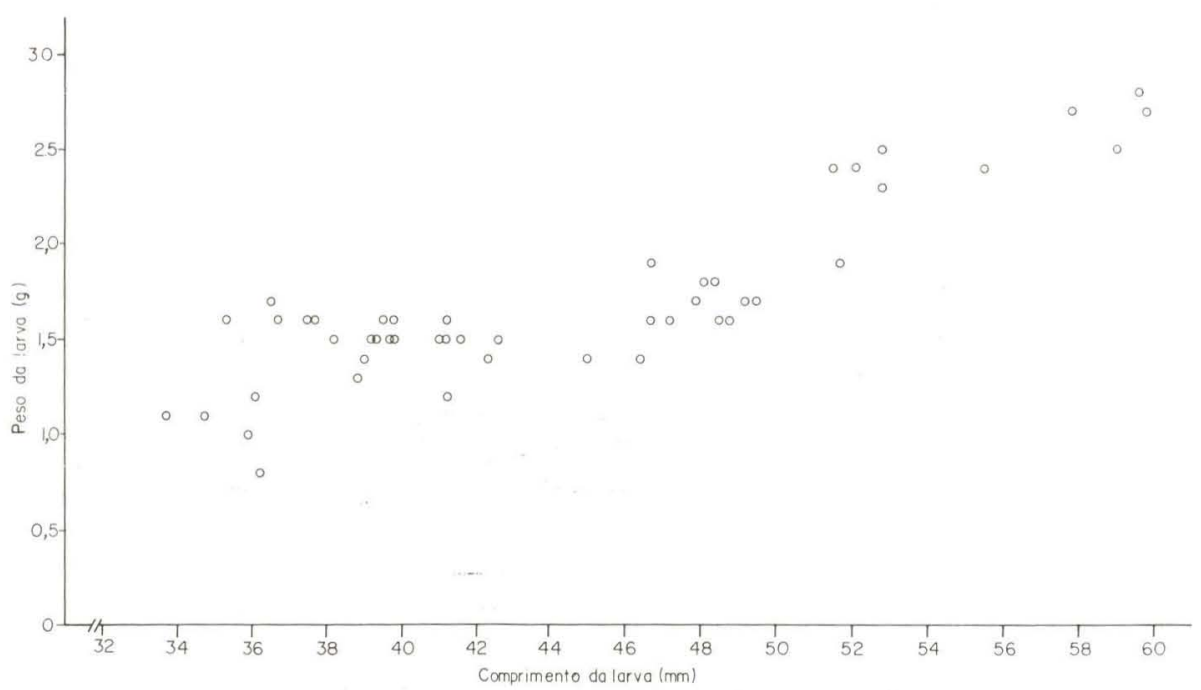

Fig. 2. Relaçāo entre os valores de peso e de comprimento em larvas de Genidens genidens. Número total de larvas mensuradas: 50. 

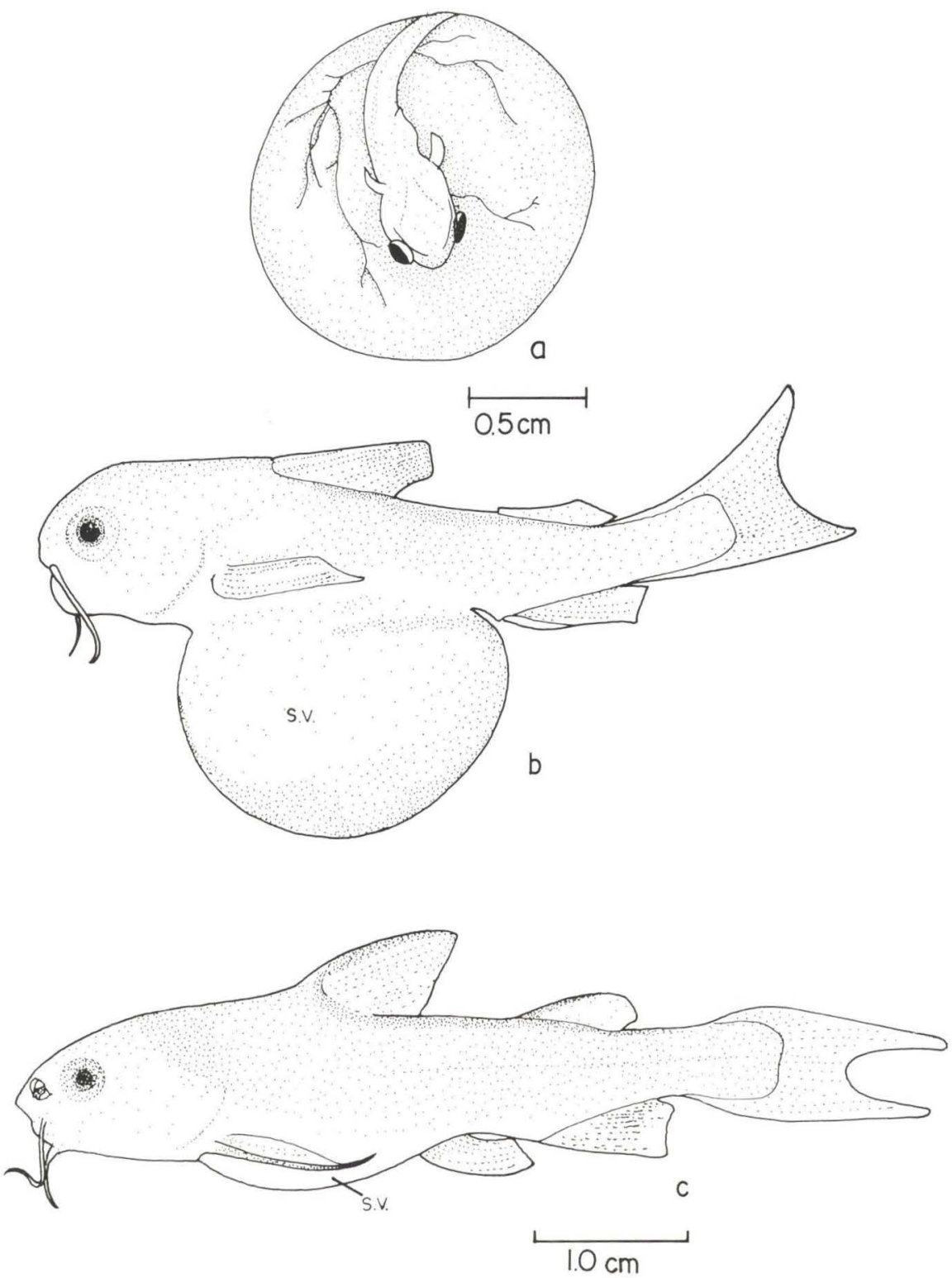

Fig. 3. Três momentos do desenvolvimento de Genidens genidens durante a incubação orofaringeana: ovo (a), larva com $32 \mathrm{~mm}$ (b) e larva com $59 \mathrm{~mm}$ (c). Observar a progressiva redução do saco vitelínico (S.V.).

Revta bras. Zool. 11 (4): 641 - 648, 1994 
água demonstraram não apenas habilidade natatória, mas também de abertura bucal e movimentação opercular. A análise do estômago das larvas revelou que $100 \%$ continham em seu interior itens de origem exógena, como fitoplâncton, microcrustáceos, fragmentos minerais e restos vegetais macroscópicos. Observou-se ainda a presença de sementes ou frutos de forma esférica, que investigação paralela revelou serem semelhantes aos registrados na dieta de adultos não-incubadores. Em alguns exemplares, restos orgânicos da mesma natureza do conteúdo gástrico foram encontrados também no intestino e no orifício anal.

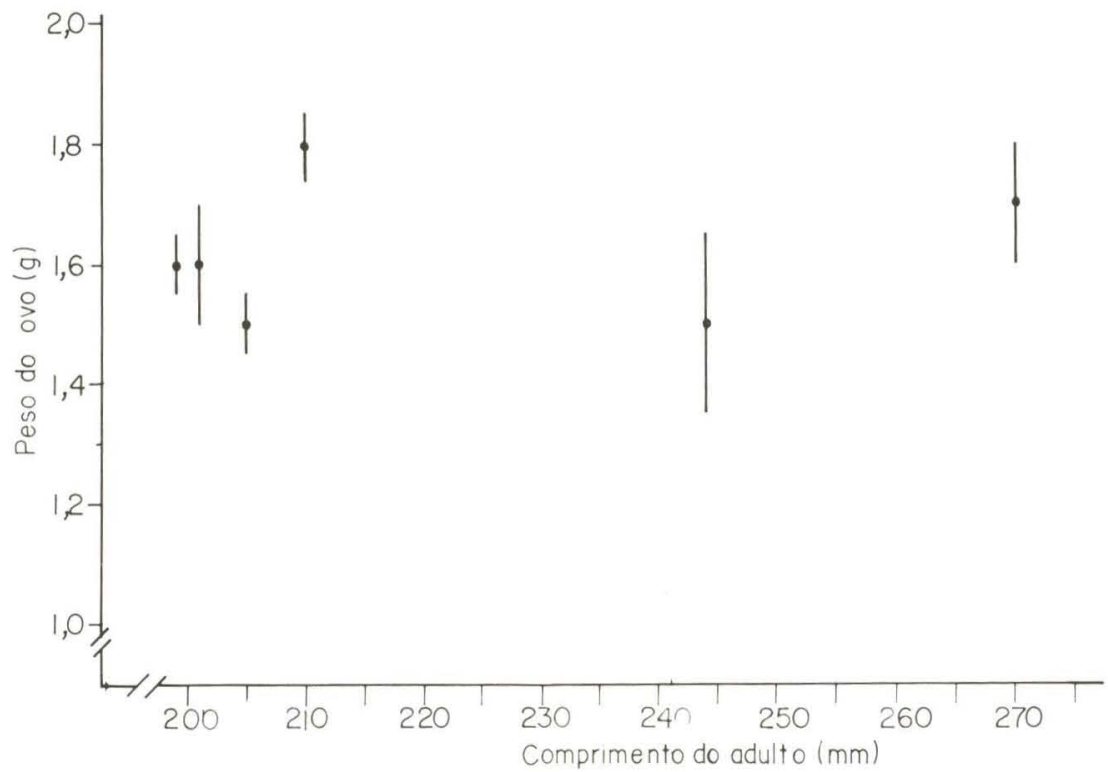

Fig. 4. Relação entre os valores de comprimento de seis adultos incuhadores e de peso dos ovos neles encontrados. São representadas as médias e as amplitudes de variação em cada exemplar.

Os adultos incubadores possuíam comprimentos entre 192 e $270 \mathrm{~mm}$. Verificou-se que o peso médio dos ovos não foi influenciado pelo tamanho do incubador (Fig. 4), mas que as larvas de maior porte sim, eram incubadas pelos adultos maiores (Fig. 5). Verificou-se ainda que o tamanho das larvas que compartilhavam uma mesma câmara orofaringeana era aproximadamente homogêneo, apresentando variação menor que quando comparados entre si os tamanhos médios de larvas procedentes de vários incubadores (Tab. I). Em um indivíduo, entre os ovos incubados havia um não-embrionado, e em outro foi registrado um ovo duplamente embrionado.

Analisadas as gônadas dos adultos incubadores, não foi possível reconhecer o sexo ou grau de maturação, visto o pequeno volume destes órgãos e a falta de um acompanhamento prolongado. Quanto ao trato digestivo, apresentou-se sempre vazio. 


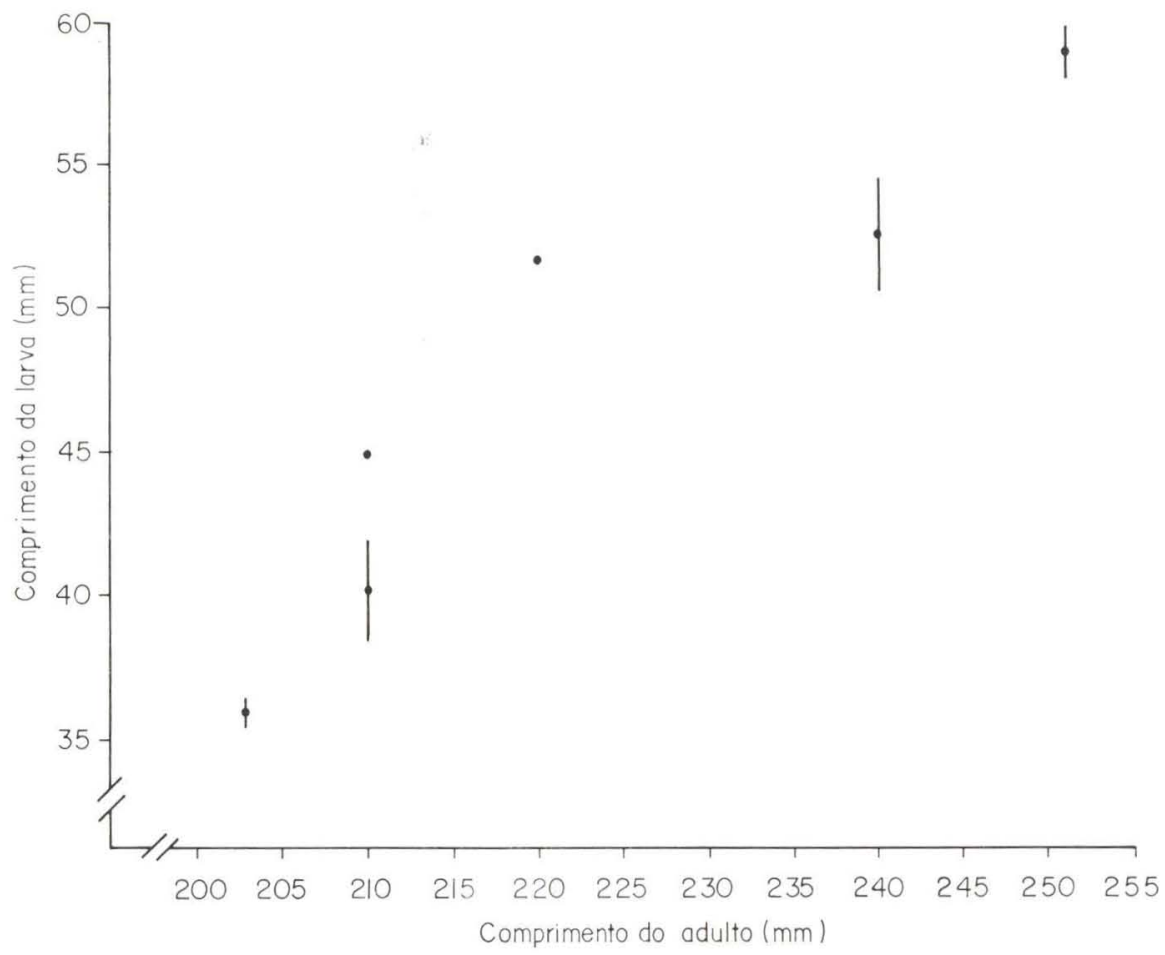

Fig. 5. Relação entre os valores de comprimento de seis adultos incubadores e de comprimento das larvas neles encontradas. São representadas as médias e as amplitudes de variação em cada exemplar.

Tabela I. Valores de comprimentos individual e médio de larvas.segundo o comprimento do adulto incubador. AMP.INT. = amplitude de variação por adulto; AMP.ENT. $=$ amplitude de variação entre as médias. Todos os dados em milímetros.

\begin{tabular}{|c|c|c|c|c|c|c|c|c|c|}
\hline \multirow[b]{2}{*}{1} & \multirow{2}{*}{$\begin{array}{l}\text { COMPRIMENTO } \\
\text { ADULTO } \\
203\end{array}$} & \multicolumn{6}{|c|}{ COMPRIMENTO LARVAS } & \multirow{2}{*}{$\begin{array}{c}\text { COMPRIMENTO } \\
\text { MÉDIO } \\
36,2\end{array}$} & \multirow{2}{*}{$\begin{array}{c}\text { AMP. INT } \\
1,4\end{array}$} \\
\hline & & 35,3 & 36,5 & 36,7 & & & & & \\
\hline 2 & 210 & 38,2 & 39,8 & 39,8 & 41,2 & 41,2 & 41,6 & 40,3 & 3,4 \\
\hline 3 & 210 & 45,0 & & & & & & 45,0 & - \\
\hline 4 & 220 & 51,7 & & & & & & 51,7 & \\
\hline 5 & 240 & 51,5 & 51,5 & 52,1 & 52.8 & 55,4 & & 52,7 & 3,9 \\
\hline \multirow[t]{2}{*}{6} & 251 & 57.8 & 59,6 & 59,7 & & & & 59,0 & 1,9 \\
\hline & & & & & & & MP. EN & 22,8 & \\
\hline
\end{tabular}

\section{DISCUSSÃO}

Os dados obtidos indicam que ao início do desenvolvimento larval o indivíduo experimenta uma redução de peso, provavelmente devida ao consumo de energia com respiração e movimentos. A seguir, porém, o peso é gradativamente recuperado (e até superado, a partir de cerca de $50 \mathrm{~mm}$ ) por alimentação exógena. 
Esta hipótese é sustentada em virtude de o peso dos ovos não ter, em nenhum caso, ultrapassado $1,9 \mathrm{~g}$, valor bem abaixo dos $2,8 \mathrm{~g}$ atingidos pelas larvas. Para a ingestão dos itens alóctones deve contribuir a circulação de água pela boca e cavidade opercular, conforme visualizado em larvas prematuramente saídas da incubação.

O tamanho exato das larvas quando da eclosão não pôde ser definido, mas é possível que seja inferior a 32,0mm (tamanho mínimo das larvas em Guaratuba), pois BARBIERI et al. (1992) encontraram em G. genidens de Jacarepaguá (Rio de Janeiro) larvas com comprimento de até $25 \mathrm{~mm}$. Por outro lado, em Guaratuba (Paraná) as larvas mantêm reserva vitelínica até pelo menos $59 \mathrm{~mm}$, valor superior ao registrado pelos mesmos autores para a população de Jacarepaguá $(55 \mathrm{~mm})$.

As larvas maiores são incubadas pelos adultos de maior porte, e sobre tal fato três alternativas podem ser propostas. Primeira, de que os adultos de grande porte incubam ovos maiores, que por isso crescem mais. Tal comportamento não pôde ser confirmado, pois desta família sabe-se apenas que as fêmeas maiores produzem ovócitos de maior tamanho (COATES 1988), e que os machos maiores incubam mais ovos (MISHIMA \& TANJI 1985; BARBIERI et al. 1992) que os menores. Ou seja, também pelo verificado em Guaratuba, incubadores de maior porte não necessariamente recebem ovos maiores que outros adultos. Uma segunda possibilidade é de que uma cavidade orofaringeana maior ofereça aos embriões mais espaço para crescimento. Esta alternativa esbarra em dados da literatura, que afirmam haver uma relação direta entre o tamanho do incubador e o número de larvas presentes na cavidade orofaringeana (BARBIERI et al. 1992). Assim, se o espaço total (e volume de água circulante) é maior, maior também é a densidade de larvas a dividi-lo, resultando provavelmente num espaço médio individual semelhante ao de incubadores de menor porte. Finalmente, uma terceira alternativa permite propor que nos adultos de maior porte as larvas sejam maiores por estarem sendo incubadas há mais tempo. De fato, é estimado que em $G$. genidens (BARBIERI et al. 1992), como também em Cathorops spixii (Agassiz, 1829) (ETCHEvERS 1978), os indivíduos maiores se reproduzem mais cedo que os menores. Assim, incubadores de maior porte coletados simultaneamente a outros, menores, devem conter larvas um pouco mais velhas, e portanto de tamanho maior.

O tamanho dos maiores adultos incubadores encontrados na baía de Guaratuba $(270 \mathrm{~mm})$ superou aquele registrado para a mesma espécie na lagoa de Jacarepaguá - 249mm - por BARBIERI et al. (1992). Esta diferença pode ser explicada tanto pelas características amostrais em cada local, como pela própria estrutura populacional, visto que o tamanho mínimo também foi menor em Jacarepaguá (162 mm, enquanto em Guaratuba 192mm).

A incubação de ovos desprovidos de embrião, como aqui verificado em um indivíduo, foi encontrada em espécies de Ariidae (REIS 1986; BARBIERI et al. 1992). Classicamente, dados da literatura informam que em Ariidae cabe ao macho a atividade incubadora (BREEDER \& ROSEN 1966), e BALON (1975) até postula que, em termos evolutivos, seja esta a condição original nos peixes. Neste trabalho, informações sobre o sexo do adulto incubador não puderam ser confirmadas. 
Colheram-se fortes evidências de que na baía de Guaratuba os adultos em processo de incubação de nada ou de pouco se alimentam, conforme já conhecido da literatura para Ariidae de outras regiões (GUNTER 1947). De fato, as observações realizadas mostraram que os ovos e as larvas obstruem completamente a cavidade orofaringeana, possivelmente apenas permitindo a passagem de água para as brânquias.

Quanto à época de ocorrência da incubação, o registro na baía de Guaratuba em meses de água mais quente, como janeiro e fevereiro, é também o caso comum descrito para a família (MISHIMA \& TANII 1985; ReIs 1986; BARBIERI et al. 1992).

AGRADECIMENTOS. Ao Conselho Nacional de Desenvolvimento Científico e Tecnológico $(\mathrm{CNPq})$ e à Fundação da Universidade Federal do Paraná, pela concessão de auxílios. Ainda ao acadêmico Dalton T.R. dos Santos, pela arte tinal das ilustrações, e a dois Revisores anônimos.

\section{REFERÊNCIAS BIBLIOGRÁFICAS}

BALON, E.K. 1975. Reproductive guilds of fishes: a proposal anddefinition. J. Fish. Res. Bd Can. 32: 821-864.

BarbieRI, L.R.; R.P. SANTOS \& J.V. AndREATA. 1992. Reproductive biology of the marine catfish, Genidens genidens (Siluriformes, Ariidae), in the Jacarepaguá Lagoon system, Rio de Janeiro, Brazil. Environ. Biol. Fishes 35 : 23-35.

Breeder JR., C.M. \& D.E. Rosen. 1966. Modes of Reproduction in Fishes. T.F.H. Publications, USA, 941 p.

COATES, D. 1988. Length-dependent changes in egg size fecundityin females, and brooded embryo size in males, of fork-tailed catfishes (Pisces: Ariidae) from the Sepik River, Papua New Guinea, with some implications for stock assessments. J. Fish Biol. 33: 455-464.

Etchevers, S.L. 1978. Contribution to the biology of the sea catfish, Arius spixii (Agassiz) (Pisces, Ariidae), south of Margarita Island, Venezuela. Bull. mar. Sci.28 (2): 381-385.

GUNTER, G. 1947. Observation on breeding of the marine catfish, Galeichthys felis (Linnaeus). Copeia 4: 217-223.

Mishima, M. \& S. TANJI. 1985. Fecundidade e incubação dos bagres marinhos (Osteichthyes, Ariidae) do complexo estuarino lagunar de Cananéia $\left(25^{\circ} \mathrm{S}\right.$, $\left.48^{\circ} \mathrm{W}\right)$. Bolm Inst. Pesca, São Paulo, 12 (2): 77-85.

Oppenheimer, J.R. 1970. Mouthbreeding in fishes. Anim. Behav. 18: 493-503. PEDA, J.C; R.M. SANChEZ \& M.M. JameTt. 1988. Desarrollo embrionario, larval y del alevín de Cichlasoma dovii (Gunther, 1864) (Pisces: Cichlidae). Revta Biol. trop. 36 (2B): 417-422.

REIS, E.G. 1986. Reproduction and feeding habits of the marine catfish Netuma barba (Siluriformes, Ariidae) in the estuary of Lagoa dos Patos, Brazil. Atlântica, Rio Grande, 8: 35-55.

Recebido em 10.V.1994; aceito em 08.VIII.1994. 